\title{
Management of retroperitoneal liposarcoma: A case report and review of the literature
}

\author{
WEI-DONG ZHANG ${ }^{*}$, DA-REN LIU*, RI-SHENG QUE, CHUAN-BIAO ZHOU, \\ CHEN-NI ZHAN, JIAN-GANG ZHAO and LI CHEN
}

\begin{abstract}
Department of Surgery, The Second Affiliated Hospital, College of Medicine, Zhejiang University, Hangzhou, Zhejiang 310009, P.R. China
\end{abstract}

Received July 27, 2014; Accepted April 21, 2015

DOI: $10.3892 / 01.2015 .3193$

\begin{abstract}
Retroperitoneal liposarcoma is a rare tumor with an incidence of 2.5 per million individuals. Early diagnosis is difficult as there is an absence of specific clinical presentations. The present case study reports a patient diagnosed with retroperitoneal liposarcoma who was treated by complete surgical resection and relapsed 3 months following the surgery. In addition, the clinical data of 14 patients with retroperitoneal liposarcoma were reviewed and analyzed. The mean age of the 14 patients at presentation was 54.1 (range, 36-73 years) and 5/14 patients experienced recurrence, ranging between 1 and 10 times. Of the 12 cases that reported histological subtypes, 7 were well-differentiated liposarcoma, 2 were dedifferentiated liposarcoma, 2 were myxoid liposarcoma and 1 was mixed subtype. All the patients underwent complete resection and 5 received combined multiple organs resection (3 nephrectomy, 1 sigmoid colon and 1 multiple visceral organs). However, no patients received chemotherapy or radiotherapy. In conclusion, retroperitoneal liposarcoma is a rare disease with a high rate of recurrence. Complete resection is the predominant treatment and combined resection of adjacent organs is occasionally necessary.
\end{abstract}

\section{Introduction}

Liposarcoma is a common type of soft tissue sarcoma (1), which occurs most commonly in the extremities (52\%), followed by the retroperitoneum (19\%) (2). Retroperitoneal liposarcoma is usually asymptomatic until the liposarcoma is large enough to compress the surrounding organs (3). It is often

Correspondence to: Professor Li Chen, Department of Surgery, The Second Affiliated Hospital, College of Medicine, Zhejiang University, 88 Jiefang Road, Hangzhou, Zhejiang 310009, P.R. China E-mail: chenli@mail.hz.zj.cn

*Contributed equally

Key words: retroperitoneal liposarcoma, resection, recurrence misdiagnosed due to its rarity and absence of symptoms. In the experience of the authors, symptoms would only occur if the liposarcoma presses on the surrounding organs. However, the retroperitneum is a large space in which the retroperitoneal liposarcoma to grow. The symptoms of the tumor would not arise until the tumor grows to a certain dimension. The management is surgical intervention. Even with complete removal of the liposarcoma, prognosis remains poor. The 5 -year survival rate of well-differentiated retroperitoneal liposarcoma is $83 \%$, while it is $20 \%$ for the dedifferentiated tumor subtype (4). Successful complete resection of retroperitoneal liposarcoma may increase the 5-year survival rate. To the best of our knowledge, there is currently no evidence that chemotherapy or radiotherapy improve survival rates. The current study presented a patient diagnosed with retroperitoneal liposarcoma who was treated in April 2014. In addition, 13 cases of retroperitoneal liposarcoma from the Chinese literature were also reviewed. Recurrence was observed in $5 / 14$ patients. All the patients underwent complete resection and 5 received combined multiple organs resection. The aim of the present study was to report a giant retroperitoneal liposarcoma, and to discuss the epidemiology, histopathology, imaging characteristics and the management of retroperitoneal liposarcoma. Approval for the present study was obtained from the ethics committee of Zhejiang University (Hangzhou, China) and informed consent was provided by the patient.

\section{Case report}

A 48 year-old female was admitted to the Department of Surgery, The Second Affiliated Hospital, College of Medicine, Zhejiang University (Hangzhou, China) in April 2014, presenting with abdominal pain in the left side for one month, accompanied by abdominal distention following eating. The past medical and surgical history of the patient had no relevance to the case. The physical examination indicated a $25 \times 35 \mathrm{~cm}$ oval mass with medium texture below the left costal margin without tenderness or rebound tenderness. The laboratory examinations, including routine blood and urine tests. CA199, 10.8 U/ml (normal range, $<37 \mathrm{U} / \mathrm{ml}$ ); CA153 7.5 U/ml (normal range, $<30 \mathrm{U} / \mathrm{ml}$ ); CA125 $5.4 \mathrm{U} / \mathrm{ml}$ (noraml range, $<35 \mathrm{U} / \mathrm{ml}$ ); CEA $1.1 \mathrm{ng} / \mathrm{ml}$ (normal range, $<5 \mathrm{ng} / \mathrm{ml}$ ); and AFP, 




Figure 1. Contrast-enhanced abdominal CT of the patient. Primary liposarcoma: CT demonstrated a giant mass in the left abdomen, pressing into the left kidney (the white arrow points to the primary liposarcoma).

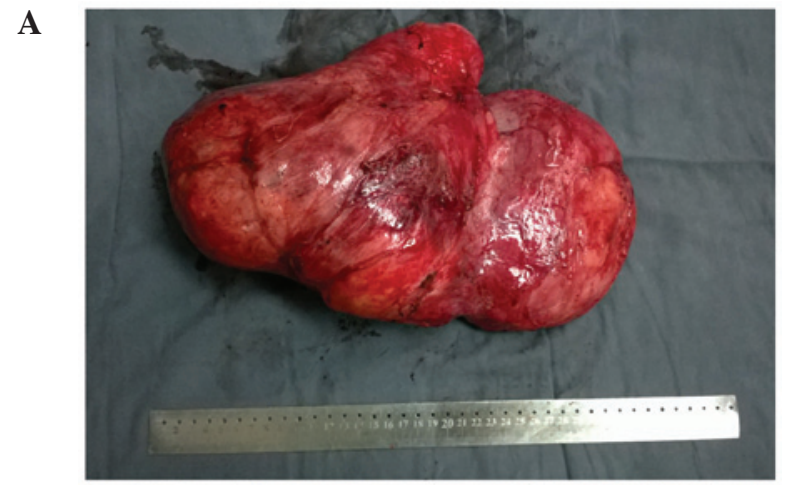

B

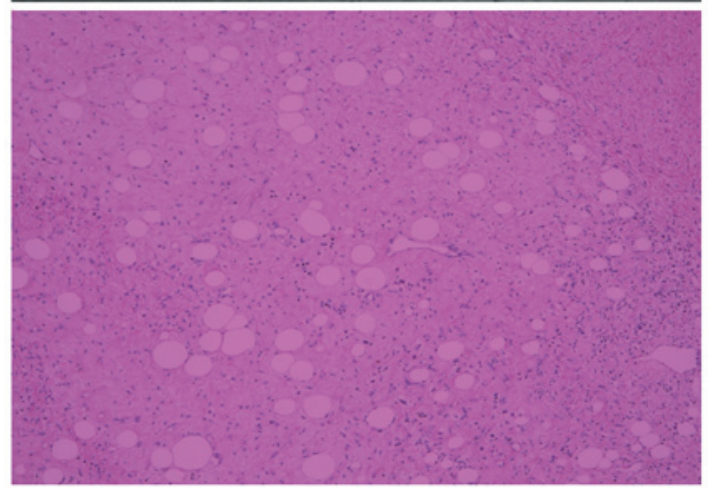

Figure 2. Macroscopic and microscopic view of the tumor. (A) Gross view of the liposarcoma: 30x20x15 $\mathrm{cm}$ in size. (B) Histological examination findings: The tumor was composed predominantly of myxoid stroma and of small amounts of mature lipocyte-like cells that were supported by rich thin arborizing vasculature and delicate fibrous septa (hematoxylin and eosin; magnification, $\mathrm{x} 50$ ).

$0.9 \mathrm{ng} / \mathrm{ml}$ (normal range, $<20 \mathrm{ng} / \mathrm{ml}$ ). All the results were within the reference ranges and therefore normal. Routine blood test: Red blood cell count, $4.51 \times 10^{12} / 1$ (normal range, $3.5-5.0 \times 10^{12} / 1$ ); white blood cell count, $6.6 \times 10^{9} 1$ (normal range: $4.0-10.0 \times 10^{9} / 1$ ); platelet count, $198 \times 10^{9} / 1$ (normal range, 100-300x10 $/ 1$ ); hemoglobin, $121 \mathrm{~g} / 1$ (normal range, 110-150 g/l). Routine urine test: Urine specific gravity, 1.013 (normal range, 1.010-1.030); $\mathrm{pH} 5.5$ (normal range: $\mathrm{pH}$ 5.0-7.0); red blood cell count, $0 / \mathrm{HP}$ (normal range, 0-3/HP); white blood cell count, 1/HP (normal range, $0-5 / \mathrm{HP}$ ). Computed tomography (CT; Fig. 1) and magnetic resonance imaging (MRI) demonstrated a giant

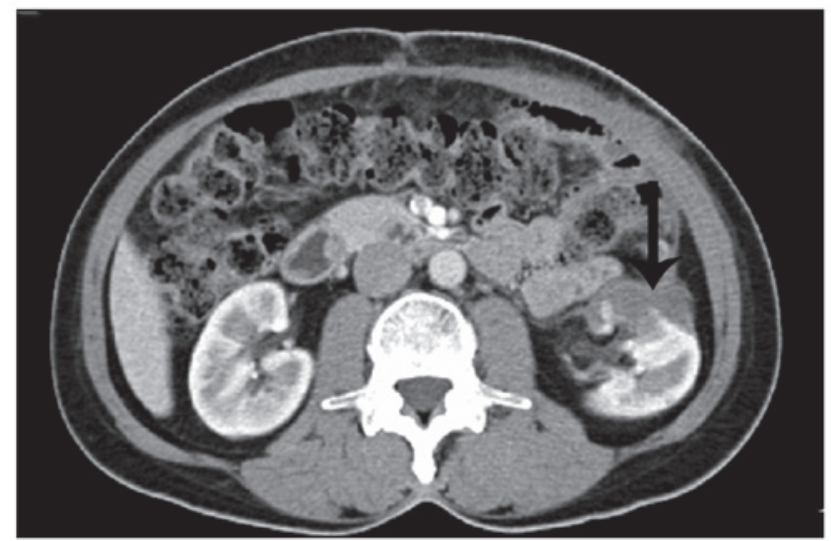

Figure 3. Contrast-enhanced abdominal CT of the patient. Recurrent liposarcoma: CT demonstrated a recurrent liposarcoma (black arrow). CT, computed tomography.

mass in the left abdomen, pressing into the left kidney and pancreas; therefore, it was decided surgery was necessary.

During surgery, it was determined that the mass originated from the left fatty renal capsule and the kidney was partly invaded. The patient received complete resection of the liposarcoma and partial resection of the left kidney. The mass was $30 \times 20 \times 15 \mathrm{~cm}$ in size (Fig. 2A).

The mass was pathologically identified as myxoid liposarcoma (Fig. 2B). The immunohistochemical analysis was conducted at the Department of Pathology at The Second Affiliated Hospital of the College of Medicine, Zhejiang University (Hangzhou, China), and revealed that the mass was positive for vimentin, weakly positive for S-100 and negative for cytokeratin (AE1/AE3). A few tumor cells were positive for the proliferation marker Ki-67. The patient recovered well and was discharged 1 week following surgery. However, 3 months following the surgery, recurrence was observed in the remainder of the left kidney (Fig. 3).

The Chinese Biology and Medicine Database (sinomed. imicams.ac.cn/zh), the Chinese periodical Database of Science and Technology (lib.cqvip.com) and the China Hospital Knowledge Database (www.chkd.cnki.net) were searched for historical cases of retroperitoneal liposarcoma between January 2005 and December 2014. Duplicate reports were excluded and 13 cases along with the 1 case treated in the current study were identified (Table I). Of the 8 cases with follow-up, none have succumbed to the disease.

The mean age of the 14 patients at presentation was 54.1 years (range, 36-73 years) and the male/female ratio was 2:5. CT was the most common auxiliary examination used for diagnosis (12/13), followed by MRI and ultrasonography. No cases were diagnosed using preoperative biopsy. Recurrence was observed in 5/14 patients, ranging between 1 and 10 times. The liposarcoma size ranged between $8 \times 8 \times 8$ and $43 \times 40 \times 25 \mathrm{~cm}$. Of the 12 cases with a reported histological subtype, 7 were well-differentiated, 2 were dedifferentiated, 2 were myxoid and 1 was of a mixed subtype. All the patients underwent complete resection, of whom 5 received additional visceral organ resection (3 nephrectomy, 1 sigmoid colon and 1 multiple visceral organ resection). However, no patients received chemotherapy or radiotherapy. 


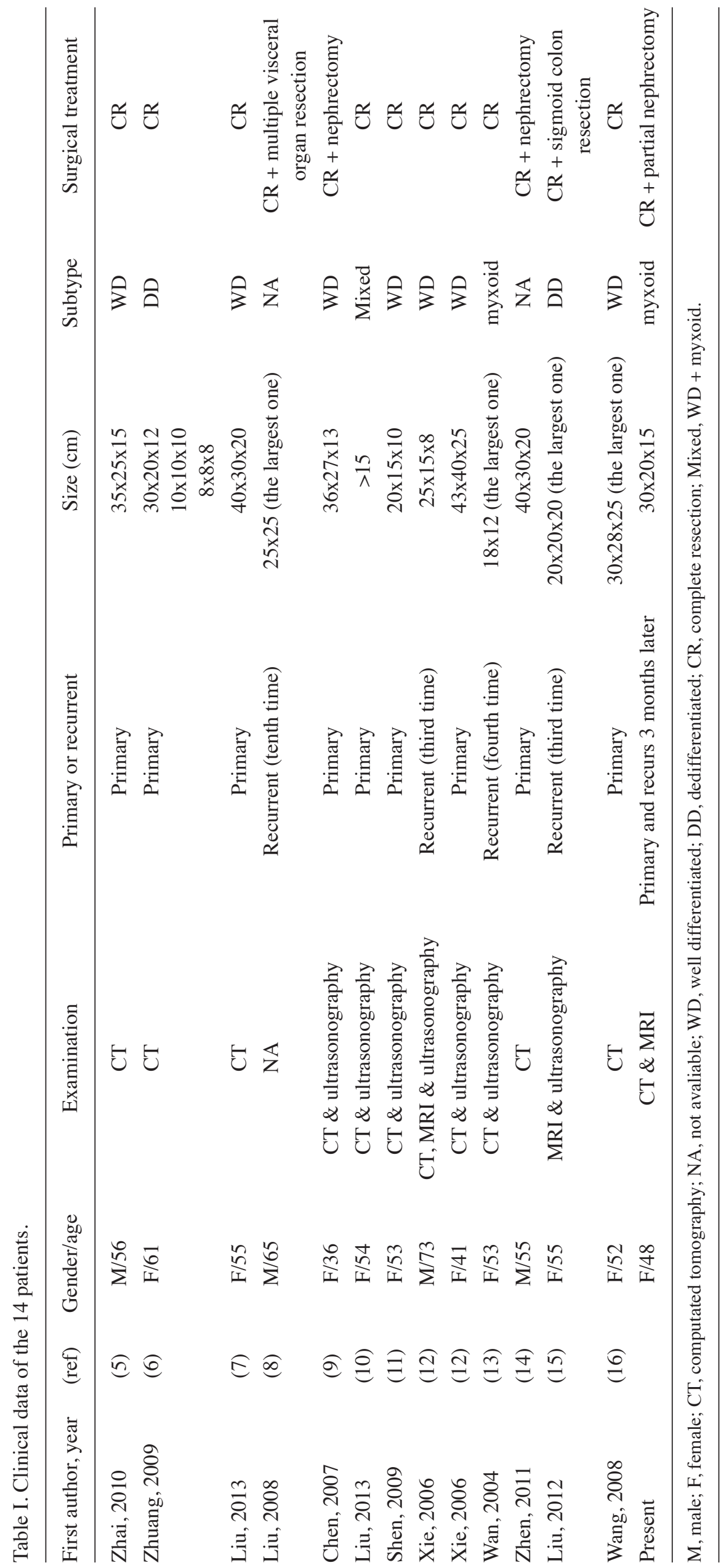




\section{Discussion}

Retroperitoneal tumor predominantly originates from fat, loose connective tissue, fascia, muscles, lymphatic tissue or residual embryonic tissue, of which $80 \%$ is malignant. Retroperitoneal liposarcoma is the most frequently observed subtype of retroperitoneal tumor, with an incidence of $\sim 2.5$ per million individuals (17). It usually occurs at 40-60 years of age, and the ratio between the genders is $\sim 1: 1(1,18)$. In the review of the literature for the present study, the mean age of the patients was 54.1 years.

The large volume of the intra-abdominal space allows liposarcoma to grow without compressing the vital organs, resulting in rare early diagnosis. When clinical symptoms do present, the retroperitoneal liposarcoma has usually grown very large in size, oppressing or invading the surrounding organs.

To differentiate from other soft tissue tumors, auxiliary examinations are required, including ultrasonography, CT and MRI. These examinations provide information on the tumor's position and size, in addition to an appropriate staging of the tumor extension and involved structures, which aids greatly in designing a surgical scheme. Ultrasonography is usually selected prior to CT scan and MRI for its convenience. On a CT scan, retroperitoneal liposarcoma usually appears as a large encapsulated mass containing variable amounts of fatty and soft tissue components (19). MRI is important for the diagnosis of liposarcoma invasion of the abdominal aorta or inferior vena cava. Biopsy is not generally recommended due to the probability of tumor seeding (20). In the present study, the literature review reported that 12 patients received CT scans for diagnosis in the 13 cases with available auxiliary examinations data. Ultrasonography and MRI were also selected in a number of cases. No cases used preoperative biopsy for diagnosis.

The final diagnosis of retroperitoneal liposarcoma is dependent on the pathological and immunohistochemical analyses. Liposarcoma can be histologically subdivided into 5 subtypes: Well-differentiated, myxoid, round cell, pleomorphic and dedifferentiated (21). Generally, round cell, pleomorphic and dedifferentiated subtypes are regarded as high-grade; whilst well-differentiated and myxoid liposarcoma are low-grade (22). With the development of pathological analysis, greater importance has been attached to the subtypes of retroperitoneal liposarcoma. High histological grade is one of the most important negative prognostic factors in patients with retroperitoneal liposarcoma. Well-differentiated liposarcomas may recur locally, but the metastatic potential is low, while pleomorphic liposarcomas have high metastatic potential, which may reduce the survival rate (22).

With regards to the high recurrence rate of liposarcoma, the standardization of the original surgery becomes particularly important. Complete resection is the predominant treatment. The principle of the surgery is to resect the tumor and any invaded organs without any residue of the liposarcoma or membrane remaining. Successful complete resection of retroperitoneal liposarcoma may increase the 5-year survival rate from 16.7 to $58 \%$ (23). However, complete resection is a challenge, particularly in the well-differentiated subtype, for the reason that the margins are not easily distinguishable (24).
Combined resection is occasionally required to achieve macroscopic clearance; the kidney is the most commonly removed organ, followed by the colon (24). In the present study, all the patients underwent complete resection, of whom 5 received additional visceral organ resection (3 nephrectomy, 1 sigmoid colon and 1 multiple visceral organs). In the present case, the liposarcoma invaded the left renal capsule and was completely macroscopically removed. Regardless, recurrence was observed. This raises the question: Should the management of the liposarcoma in the present case have been more aggressive? Should the whole kidney be resected rather than the renal capsule and part of the kidney (if the renal function of the contralateral kidney is sufficient)? Certain previous studies support the above argument and indicate that if the liposarcoma invaded the kidney, then combined resection of the kidney is necessary $(9,25)$. There is no evidence that chemotherapy or radiotherapy improve survival rates (26). In the present study, no patients in the studied literature were administered chemotherapy or radiotherapy.

Even with complete removal of the liposarcoma, prognosis remains poor. The 5-year survival rate of well-differentiated retroperitoneal liposarcoma is $83 \%$, while it is $20 \%$ for the dedifferentiated tumor subtype (22). The total recurrence rate was $5 / 14$ patients in the present literature review and the recurrence frequency ranged between 1 and 10 times. Local recurrence remains the preliminary cause of mortality in retroperitoneal liposarcoma (27). The survival rate was improved in the patients who received a complete resection of their recurrent tumor compared with the patients who did not. Therefore, the gold standard treatment remains to be removal of the recurrence (25). The difficulty of the secondary operation is that the anatomical relationship would be more complex. The reason for this is that after the initial surgery, the anatomical position would have altered and the tissues may adhere to one another or to important vessels, resulting in the increased difficulty of the secondary surgery. Combined resection of the surrounding organs may be unavoidable when attempting to achieve complete resection. The purpose of the secondary operation is to remove the tumor, in addition to relieving the compression of vital viscera. When radical surgery is not possible, palliative resection is advisable. In order to detect recurrence, a CT scan every 3 months for the first 2 years, every 6 months for 2-5 years and annually thereafter is generally recommended.

In conclusion, retroperitoneal liposarcoma is a rare disease with a high rate of recurrence. Complete resection is the benchmark for treatment, however the combined resection of adjacent organs is occasionally necessary. The present study reports a case of giant retroperitoneal liposarcoma and puts it into context with previously reported cases in the literature; in addition to the authors experience of the surgery, in order to summarize the standard therapy.

\section{References}

1. Mendenhall WM, Zlotecki RA, Hochwald SN, Hemming AW, Grobmyer SR and Cance WG: Retroperitoneal soft tissue sarcoma. Cancer 104: 669-675, 2005.

2. Russell WO, Cohen J, Enzinger F, et al: A clinical and pathological staging system for soft tissue sarcomas. Cancer 40: 1562-1570, 1977. 
3. Bradley JC and Caplan R: Giant retroperitoneal sarcoma: A case report and review of the management of retroperitoneal sarcomas. Am Surg 68: 52-56, 2002

4. Fabre-Guillevin E, Coindre JM, Somerhausen NS, Bonichon F, Stoeckle E and Bui NB: Retroperitoneal liposarcomas: follow-up analysis of dedifferentiation after clinicopathologic reexamination of 86 liposarcomas and malignant fibrous histiocytomas. Cancer 106 2725-2733, 2006

5. Zhai HX, Ma XM and Jia QQ: A case report of giant retroperitoneal liposarcoma. Chin Community Doct 12: 167-168, 2010 (In Chinese)

6. Zhuang GY, Dong CW, Li JQ and Li MJ: Multiple retroperitoneal dedifferentiated liposarcoma: a case report. Chin J Curr Adv Gen Surg 12: 89-90, 2009 (In Chinese).

7. Liu B, Wang QF, Yu JH, Wang DG, Zhang Y and Xu YC: Giant abdominal liposarcoma: a case report. Chin J Gerontol 33: 452, 2013 (In Chinese).

8. Liu CL and Zhang HY: Multiple retroperitoneal and abdominal liposarcoma: a case report. Chin J Gen Surg 23: 320, 2008 (In Chinese).

9. Chen RF and Peng DT: Giant retroperitoneal liposarcoma: a case report. Chin J Dig Surg 6: 380, 2007 (In Chinese).

10. Liu CZ, Xin H, Li GD and Wang LL: Giant retroperitoneal liposarcoma: a case report. Jilin Med J 34: 5535-5536, 2013 (In Chinese)

11. Shen Y, He XJ, Li Y, Lv RG and Li P: Giant retroperitoneal liposarcoma: a case report. The Journal of Practical Medicine 25: 516, 2009 (In Chinese)

12. Xie M, Zeng QL and Wen KM: Giant retroperitoneal liposarcoma: experiences in diagnosis and treatment of two cases. Guizhou Med J 30: 30-31, 2006 (In Chinese)

13. Wan HM, Li LS, Zhang G and Wang ZY: Resection of giant retroperitoneal liposarcoma for the 4th time: a case report. Chin J Prac Surg 24: 436, 2004 (In Chinese).

14. Zheng SW, Wang SL and An WK: Giant retroperitoneal liposarcoma: a case report. Clin J Med Offic 39: 333, 2011 (In Chinese)

15. Liu J, Bai YC, Zhang YX, Sun Y and Zhang MZ: Multiple giant retroperitoneal liposarcoma: a case report. Prac J Med \& Pharm 29: 292, 2012 (In Chinese).
16. Wang XH and Wang HG: Giant retroperitoneal liposarcoma. Chin Med Her 5: 118, 2008 (In Chinese).

17. Neuhaus SJ, Barry P, Clark MA, Hayes AJ, Fisher C and Thomas JM: Surgical management of primary and recurrent retroperitoneal liposarcoma. Br J Surg 92: 246-252, 2005.

18. Mack TM: Sarcomas and other malignancies of soft tissue, retroperitoneum, peritoneum, pleura, heart, mediastinum, and spleen. Cancer 75 (Suppl): 211-244, 1995.

19. Chang IY and Herts BR: Retroperitoneal liposarcoma. J Urol 189: 1093-1094, 2013.

20. Clark MA and Thomas JM: Portsite recurrence after laparoscopy for staging of retroperitoneal sarcoma. Surg Laparosc Endosc Percutan Tech 13: 290-291, 2003.

21. Singer S, Antonescu CR, Riedel E and Brennan MF: Histologic subtype and margin of resection predict pattern of recurrence and survival for retroperitoneal liposarcoma. Ann Surg 238: 358-371, 2003.

22. Nijhuis PH, Sars PR, Plaat BE, Molenaar WM, Sluiter WJ and Hoekstra HJ: Clinico-pathological data and prognostic factors in completely resected AJCC stage I-III liposarcomas. Ann Surg Oncol 7: 535-543, 2000.

23. Lee SY, Goh BK, Teo MC, et al: Retroperitoneal liposarcomas: the experience of a tertiary Asian center. World J Surg Oncol 9: $12,2011$.

24. Chen ZH and Song XM: The theraputic progress in retroperitoneal liposarcoma. Chin J Gen Surg 24: 81-83, 2009 (In Chinese).

25. Bautista N, Su W and O'Connell TX: Retroperitoneal soft-tissue sarcomas: Prognosis and treatment of primary and recurrent disease. Am Surg 66: 832-836, 2000.

26. Perez EA, Gutierrez JC, Moffat FL Jr, et al: Retroperitoneal and truncal sarcomas: prognosis depends upon type not location. Ann Surg Oncol 14 :1114-1122, 2007.

27. Milone M, Pezzullo LS, Salvatore G, Pezzullo MG, Leongito M, Esposito I and Milone F: Management of high-grade retroperitoneal liposarcomas: personal experience. Updates Surg 63: $119-124,2011$ 